\title{
Gene Expression Profiling of the Mouse Pancreas during the Secondary Transition in the Organogenesis of the Pancreatic Gland*
}

\author{
Stefanie J. Willmann \\ TUM, Munich, Germany \\ Email: SJWillmann@gmx.de
}

How to cite this paper: Willmann, S.J. (2021) Gene Expression Profiling of the Mouse Pancreas during the Secondary Transition in the Organogenesis of the Pancreatic Gland. Journal of Diabetes Mellitus, 11, 1-9. https://doi.org/10.4236/jdm.2021.111001

Received: November 23, 2020

Accepted: January 31, 2021

Published: February 3, 2021

Copyright $\odot 2021$ by author(s) and Scientific Research Publishing Inc. This work is licensed under the Creative Commons Attribution International License (CC BY 4.0).

http://creativecommons.org/licenses/by/4.0/

\begin{abstract}
Diabetes mellitus is a chronic disease that impacts the homeostasis of blood sugar levels caused by loss or defect of insulin-producing $\beta$-cells in the Islets of Langerhans. Type 1 diabetes $(T 1 D)$ is caused by auto-immune mediated destruction of $\beta$-cells, whereas in $T 2 D$, insulin is produced but used inefficiently. T2D accounts for $90 \%$ of people with diabetes worldwide ( $W H O 1999$ ) and is the fastest increasing disease worldwide (https://diabetesatlas.org/en/). For an improved understanding of the pathomechanism of diabetes, profound knowledge of pancreas organogenesis and the associated gene regulatory networks is required. Therefore, we dissected and profiled the pancreatic endodermal and non-endodermal compartment between the embryonic stages (E) 12.5 and E 15.5 when progenitor cells commit to their different pancreatic lineages. Our associated study mined the global mRNA expression profile to increase the understanding of the secondary transition, endodermal-non-endodermal tissue interaction, and diabetic-related gene regulation. Furthermore, we validated 635 regulated pancreatic genes using the publicly available GenePaint.org, respective gp3.mpg.de to evaluate genes associated with genetic variants in Single-nucleotide polymorphism (SNP) related to $T 2 D$.
\end{abstract}

\section{Keywords}

Gene Expression Profile, Pancreas Organogenesis, Single-Nucleotide Polymorphism, Type 1 Diabetes, Type 2 Diabetes

*The author thanks the people as follows for the great work together: Nikola S. Mueller, Michael Sterr, Ingo Burtscher, Aurelia Raducanu, Silvia Engert, Martin Irmler, Johannes Beckers, Steffen Sass, Fabian J. Theis, Heiko Lickert. 


\section{Experimental Design, Materials and Methods}

\subsection{Dissection, Isolation, and Processing}

Pancreatic tissue was isolated from FVF+ embryos at E 12.5 to E 15.5 in triplicates [1]. A single-cell suspension was obtained by trypsinization with $0.25 \%$ Trypsin and the specific incubated pancreatic tissue resuspension. Fluorescent-Activated cell sorting (FACs) was used to separate the FVF+ endodermal and FVF- non-endodermal cells. Total RNA was isolated using Trizol (Qiagen) and purified by using the RNeasy Mini kit (Qiagen) according to the manufacturer's protocol. The Agilent 2100 Bioanalyzer had assessed RNA quality. The total RNA of three biological replicates for each embryonic stage was used to generate cRNA by two rounds of transcription. In general, a poly (dT) primer was utilized to synthesize cDNA from total RNA. In the next step, the cDNA amplified cRNA through T7 polymerase, using the T7 promoter sequence of the poly (dT) primer in the first round by random priming. Next, the cDNA was employed for the transcription into biotinylated cRNA, which hybridized to the 169 Affymetrix $x^{\circ}$ array format according to the manufacturer protocol (GeneChip ${ }^{\otimes}$ Gene mouse 1.0 ST Array Card). Subsequently, processing of the Fluidics script FS450_0007 and scanning (GeneChip ${ }^{\otimes}$ Scanner 3000 7G Whole-Genome Association System) further lead to the probe set outcome.

The Affymetrix ${ }^{\circ}$ Expression console normalized the robust multichip-analyses (RMA) on gene-level (ratio > 200 compared endodermal and non-endodermal). Comprehensive $\mathrm{R}$ based microarray analyses web frontend (CARMAweb) inducted gene-wise testing by Limma t-test and Benjamini-Hochberg multiple testing correction with a false discovery rate (FDR $<5 \%)$ offered statistically significant differential expression of 2921 probe sets [2]. Data were submitted to the Gene Expression Omnibus (GSE66856).

\subsection{Gene ontology (G0) Term Analysis and Validation of Gene Expression by Gene Paint in Silico in Situ}

Subsequently, GO term enrichment, annotated through association of InterPro records with GO term, grouped 635 genes in the following clusters: extracellular/located at plasma membrane (304 genes), signaling molecules (222 genes), transcription factors (TF; 178 genes), cilium related (40 genes) and previously not described (42 genes). Afterwards, pancreatic-specific gene expression and localization were analyzed using the database GenePaint.org, respective gp3.mpg.de, which offers in silico in situs of whole mount embryosat E1 4.5. Pancreatic genes had been arranged according to their expression pattern in mesenchyme (non-epithelial), tip (exocrine), trunk (endocrine) and epithelium (pancreatic multipotent cells).

\subsection{Analysis of Mouse Orthologous Genes with Associated Single-Nucleotide Polymorphism (SNP) in T1D and T2D GWAS Hits}

SNP of genes associated with $T 1 D$ and $T 2 D$ had been analyzed for their expres- 
sion level during secondary transition and depicted in a heatmap (Figure 1). The heatmap represents the relative fold change in gene expression during the secondary transition stage between the non-endodermal and endodermal tissue compartments. The clustering of the genes was accomplished through extraction of gene symbols by Marullo et al. and Hakonarson et al. [3] [4]. Based on gene expression levels in the two tissue compartments we identified different clusters of the selected genes: transcriptionally regulated in both tissue populations or elevated either in the endodermal or the non-endodermal tissue compartment.

Heatmap shows clustering of $153 T 1 D$ - and $T 2 D$-associated genes according to their expression level during lineage commitment between E12.5 - 15.5. Predominantly, maturity-onset diabetes of the young (MODY) genes are upregulated in the endodermal compartment during pancreas organogenesis ( $G c k$, Pdx1, NeuroD, Hnfla, Hnf1B). The black arrow marks the gene Ins2.

A subset of these $T 2 D$-associated genes which are regulated in our mRNA expression profile is illustrated in Table 1. Additional information of the mRNA localization (endodermal/non-endodermal), gene locus, chromosomal localization, the associated SNP and the disease as well as the related publications is provided as follows [4]-[12].

The GenePaint.org, respective gp3.mpg.dein silico in situ platform confirmed expression of mouse orthologues with associated SNPs in T2D shown in Table 1. Additional information about mRNA localization of the selected genes is obtained by inspection of the in situ whole mount embryos (see Figure 2).

Table 1. Genes associated with SNP in GWAS studies and related to T1D or T2D.

\begin{tabular}{|c|c|c|c|c|c|}
\hline \multicolumn{6}{|c|}{ Genetic variants associated with T2D or glycemic traits } \\
\hline Localization & Locus name & Chromosome & associated SNP & established loci & Literature \\
\hline non- and endodermal & TCF7L2 & 10 & $\begin{array}{l}\text { rs } 4506565, \text { rs7903146, } \\
\text { rs12243326 }\end{array}$ & T2DM & $\begin{array}{l}\text { Dupuis et al., 2012; } \\
\text { Saxena et al., } 2010\end{array}$ \\
\hline endodermal & KCNQ1 & 11 & rs 2237892, rs 231362 & T2DM & $\begin{array}{l}\text { Yasuda et al., 2008; } \\
\text { Voight et al., } 2010\end{array}$ \\
\hline endodermal & PROX1 & 1 & rs340874 & $\mathrm{T} 2 \mathrm{DM}$ & Dupuis et al., 2012 \\
\hline endodermal & HNF1 $\beta$ & 17 & $\begin{array}{l}\text { rs757210, rs4430796, } \\
\text { rs7501939 }\end{array}$ & T2DM & $\begin{array}{l}\text { Pearson et al., 2007; } \\
\text { Winckler et al., 2007; } \\
\text { Wang et al., } 2014\end{array}$ \\
\hline endodermal & $\mathrm{KCNJ11}$ & 11 & rs5219, rs757110 & $\mathrm{T} 2 \mathrm{DM}$ & Gloyn et al., 2003 \\
\hline endodermal & GCK & 7 & $\begin{array}{l}\text { rs1799881, rs6975024, } \\
\text { rs4607517, rs3757840 }\end{array}$ & T2DM & $\begin{array}{l}\text { Dupuis et al., 2012; } \\
\text { Soranzo et al., 2010; } \\
\text { Scott et al., } 2012\end{array}$ \\
\hline endodermal & FOXA2 & 20 & rs6113722, rs6048205 & $\mathrm{T} 2 \mathrm{DM}$ & $\begin{array}{c}\text { Scott et al., 2012; } \\
\text { Manning et al., } 2012\end{array}$ \\
\hline endodermal & PDX1 & 13 & rs11619319, rs2293941 & $\mathrm{T} 2 \mathrm{DM}$ & $\begin{array}{c}\text { Scott et al., 2012; } \\
\text { Manning et al., } 2012\end{array}$ \\
\hline
\end{tabular}




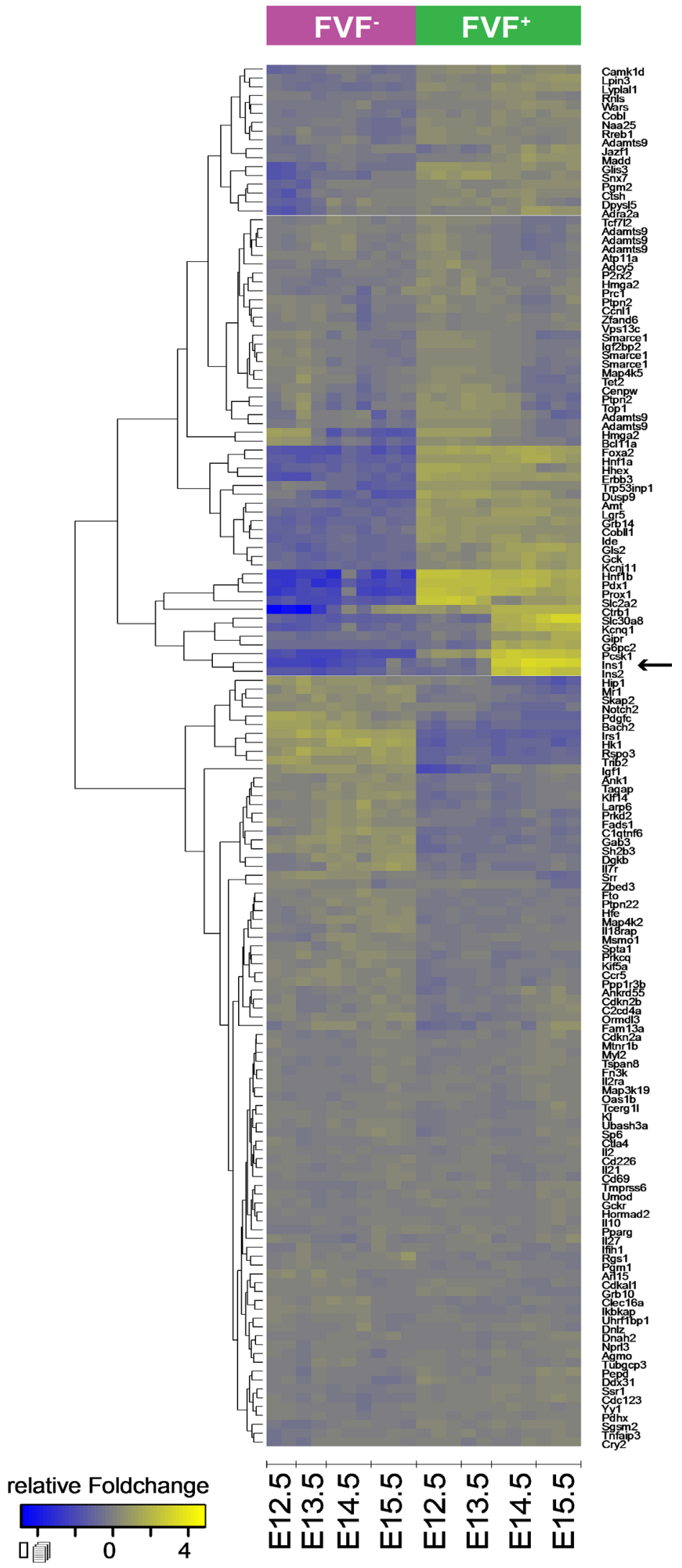

Figure 1. Heatmap depicting mus musculus orthologous genes with associated SNP in T1D and T2DGWAS hits. 

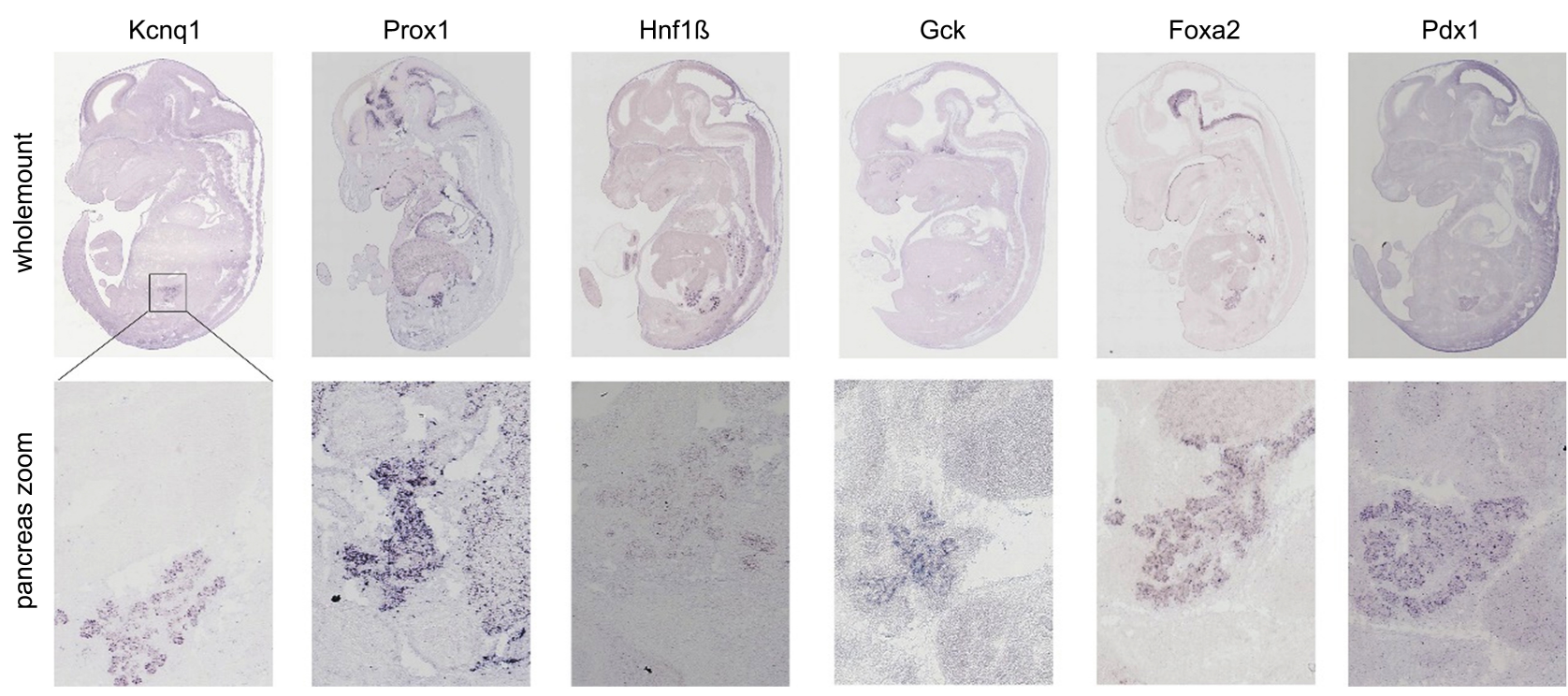

Figure 2. GenePaint in silico in situ of the genes represented in Table 1 at E14.5.

Sagittal sections of whole mount embryos and magnification of the pancreatic region are shown. The genes associated with SNP in T2D as Kcnq1, Prox1, Hnf1B, Gck, Foxa2 and $P d x 1$ exhibit epithelial in situ expression pattern.

\section{Conclusion}

The study comprehensively underpins the association of de-/regulation of developmental genes in the mouse embryo with distinct mRNA expression, validated by genepaint, respective gp3.mpg.de and genetic variants in SNP connected to the disease diabetes mellitus. Thus, the database may serve as a resource for further evaluation of mouse genes and their respective human orthologues in developmental stages and in a next step in different diseases. Further genes in the context of major health diseases as described in this study on Diabetes mellitus, Diabetes mellitus related corornary artery disease (CAD), cancers and respiratory diseases might shed light on disease mechanism and will improve therapeutic approaches for a better health outcome of the patients' need.

\section{Acknowledgements}

We thank the Helmholtz Alliance ICEMED and Helmholtz Portfolio Theme "Metabolic Dysfunction and Common Disease" (grants to HL and JB) and the European Research Council (Latent Causes: 259294; grant to FJT) for funding. Additionally, the HumEn project was financially supported from the European Union's Seventh Framework Programme for Research, Technological Development and Demonstration under grant agreement No. 602587 (http://www.cordis.europa.eu/). S.W. thanks the Helmholtz Society, German Research Foundation and German Center for Diabetes Research (DZD e.V.) for financial and arranging support and the Federal Ministry of Education and Research [Virtual Liver: FKZ 0315752 and LungSys: FKZ 0316042I] for the assistance. 


\section{Direct Link to the Deposited Data}

Data is available through the NCBI database under the following link http://www.ncbi.nlm.nih.gov/geo/query/acc.cgi?token=gnubwesiphanjiv\&acc=G SE66856.

Statistical analyses of the dataset were accomplished by using the GEO2R platform.

\section{Conflict of Interest}

The author declares that there is no conflict of interest on the work presented in the paper.

\section{References}

[1] Burtscher, I., Barkey, W. and Lickert, H. (2013) Foxa2-Venus Fusion Reporter Mouse Line Allows Live-Cell Analysis of Endoderm-Derived Organ Formation. Genesis, 51, 596-604. https://doi.org/10.1002/dvg.22404

[2] Rainer, J., Sanchez-Cabo, F., Stocker, G., Sturn, A. and Trajanoski, Z. (2006) CARMAweb: Comprehensive R- and Bioconductor-Based Web Service for Microarray Data Analysis. Nucleic Acids Research, 34, 498-503. https://doi.org/10.1093/nar/gkl038

[3] Marullo, L., El-Sayed Moustafa, J.S. and Prokopenko, I. (2014) Insights into the Genetic Susceptibility to Type 2 Diabetes from Genome-Wide Association Studies of Glycaemic Traits. Current Diabetes Reports, 14, Article Number: 551. https://doi.org/10.1007/s11892-014-0551-8

[4] Dupuis, J., Langenberg, C., Prokopenko, I., Saxena, R., Soranzo, N., Jackson, A.U., et al. (2010) New Genetic Loci Implicated in Fasting Glucose Homeostasis and Their Impact on Type 2 Diabetes Risk. Nature Genetics, 42, 105-116. https://doi.org/10.1038/ng.520

[5] Saxena, R., Hivert, M.-F., Langenberg, C., Tanaka, T., Pankow, J.S., Vollenweider, P., et al. (2010) Genetic Variation in GIPR Influences the Glucose and Insulin Responses to an Oral Glucose Challenge. Nature Genetics, 42, 142-148. https://doi.org/10.1038/ng.521

[6] Yasuda, K., Miyake, K., Horikawa, Y., Hara, K., Osawa, H., Furuta, H., et al. (2008) Variants in KCNQ1 Are Associated with Susceptibility to Type 2 Diabetes Mellitus. Nature Genetics, 40, 1092-1097. https://doi.org/10.1038/ng.207

[7] Voight, B.F., Scott, L.J., Steinthorsdottir, V., Morris, A.P., Dina, C., Welch, R.P., et al. (2010) Twelve Type 2 Diabetes Susceptibility Loci Identified through Large-Scale Association Analysis. Nature Genetics, 42, 579-589. https://doi.org/10.1038/ng.609

[8] Pearson, E.R., Donnelly, L.A., Kimber, C., Whitley, A., Doney, A.S.F., Mccarthy, M.I., et al. (2007) Variation in TCF7L2 Influences Therapeutic Response to Sulfonylureas. Diabetes, 56, 2178-2182. https://doi.org/10.2337/db07-0440

[9] Winckler, W., Weedon, M.N., Graham, R.R., McCarroll, S.A., Purcell, S., Almgren, P., et al. (2007) Evaluation of Common Variants in the Six Known Maturity-Onset Diabetes of the Young (MODY) Genes for Association with Type 2 Diabetes. Diabetes, 56, 685-693. https://doi.org/10.2337/db06-0202

[10] Gloyn, A.L. (2003) The Search for Type 2 Diabetes Genes. Ageing Research Reviews, 2, 111-127. https://doi.org/10.1016/S1568-1637(02)00061-2 
[11] Scott, R.A., Lagou, V., Welch, R.P., Wheeler, E., Montasser, M.E., Luan, J., et al. (2012) Large-Scale Association Analyses Identify New Loci Influencing Glycemic Traits and Provide Insight into the Underlying Biological Pathways. Nature Genetics, 44, 991-1005. https://doi.org/10.1038/ng.2385

[12] Manning, M.-F., Hivert, Scott, R.A., Grimsby, J.L., Bouatia-Naji, N., Chen, H., et al. (2012) A Genome-Wide Approach Accounting for Body Mass Index Identifies Genetic Variants Influencing Fasting Glycemic Traits and Insulin Resistance. Nature Genetics, 44, 659-669. https://doi.org/10.1038/ng.2274 


\section{Abbreviations}

T1D-type 1 diabetes

T2D-type 2 diabetes

WHO-world health organisation

E-embryonic stage

mRNA-messenger ribonucleic acid

SNP-single-nucleotide polymorphism

GWAS-genome-wide association study

FACs-fluorescent-activated cell sorting

FVF-Foxa2-Venus-Fusion

cDNA-complementary deoxynucleic acid

GRN-gene regulatory network

RNA-ribonucleic acid

cRNA-complementary ribonucleic acid

RMA - robust multichip-analyses

CARMA-Comprehensive R based microarray analyses web frontend

GO-gene ontology

$\mathrm{TF}$-transcription factor

\section{Specifications Table}

\begin{tabular}{|c|c|}
\hline Subject area & Biology \\
\hline More specific subject area & Developmental biology \\
\hline Type of data & Figure, Table \\
\hline How data was acquired & $\begin{array}{l}\text { Affymetrix }^{\oplus} \text { mouse gene } 1.0 \mathrm{ST} \text { array card and Affymetrix } \\
\text { Expression console. }\end{array}$ \\
\hline Data format & $\begin{array}{c}\text { CARMAweb based Limma t-test and Benjamini Hochberg } \\
\text { multiple testing corrections. }\end{array}$ \\
\hline Experimental factors & $\begin{array}{l}\text { Comparison of the mouse endodermal and non-endodermal } \\
\text { pancreatic compartment during the secondary transition } \\
\text { between E12.5 and E15.5. Bioinformatic analysis identified } \\
\text { differentially regulated genes and additionally offered the } \\
\text { narration of Genome-Wide Association Study (GWAS) } \\
\text { annotated diabetes-related genes. }\end{array}$ \\
\hline Experimental features & $\begin{array}{c}\text { Fluorescent-activated cell sorting (FACs) and RNA extraction } \\
\text { of Foxa2VenusFusion (FVF)+ endodermal and FVF- } \\
\text { non-endodermal cells. Conversion to cDNA and } \\
\text { Hybridization to the Affymetrix }{ }^{\oplus} \text { array. }\end{array}$ \\
\hline Data source location & $\begin{array}{l}\text { Garching } 85748 \text {, Germany, Am Parkring 11, } \\
\text { Institute of Diabetes Research (IDR) }\end{array}$ \\
\hline Data accessibility & $\begin{array}{l}\text { Data available at the Gene Expression Omnibus, GSE66856. } \\
\text { http://www.ncbi.nlm.nih.gov/geo/query/acc.cgi?token=gnubwesiphanjiv } \\
\underline{\text { \&acc=GSE66856 }}\end{array}$ \\
\hline
\end{tabular}




\section{Value of the Data}

- Demonstrates the value of the Foxa2-Venus fusion reporter mouse line for diabetic related research [1].

- Provides a rich data set of differentially regulated genes within the endodermal and non-endodermal tissue compartments during the secondary transition of the pancreas organogenesis.

- Offers temporal and spatial progression of mouse orthologues $T 1 D$ and $T 2 D$ associated genes.

- Evaluates database-dependent gene regulatory network (GRN) of genome-wide association studies (GWAS)-annotated diabetes genes. 